\title{
Einstein-Cartan-Heisenberg Theory of Gravity with Dynamical Torsion
}

\author{
V. Dzhunushaliev * \\ Universität Potsdam, Institut für Mathematik, 14469, Potsdam, Germany \\ and Theor. Phys. Dept. KSNU, 720024, Bishkek, Kyrgyzstan \\ D. Singleton ${ }^{\dagger}$ \\ Dept. of Phys. CSU Fresno, 2345 East San Ramon Ave. M/S 37 Fresno, CA 93740-8031, USA
}

(May 27, 2022)

\begin{abstract}
On the basis of an algebraic relation between torsion and a classical spinor field a new interpretation of Einstein-Cartan gravity interacting with a classical spinor field is proposed. In this approach the spinor field becomes an auxiliary field and the dynamical equation for this field (the Heisenberg equation) is a dynamical, gravitational equation for torsion. The simplest version of this theory is examined where the metric degrees of freedom are frozen and only torsion plays a role. A spherically symmetric solution of this theory is examined. This solution can be interpreted, in the spirit of Wheeler's ideas of "charge without charge" and "mass without mass", as a geometrical model for an uncharged particle with spin ("spin without spin").
\end{abstract}

\footnotetext{
*E-Mail Address : bars@krsu.edu.kg

${ }^{\dagger}$ E-Mail Address : das3y@maxwell.phys.csufresno.edu
} 


\section{INTRODUCTION}

The simplest generalization of general relativity is Einstein-Cartan (EC) gravitational theory in which torsion is included. Remarkably this theory is a gauge theory of gravity similar in spirit to Yang-Mills gauge theories. The difference being that the gauge group of the former is the Poincare group, while in the latter case one has gauge groups like $\mathrm{SU}(3)$ or $\mathrm{SU}(2)$. One peculiarity of Einstein-Cartan theory is that the torsion does not have a dynamical term in the Lagrangian and is therefore a nonpropagating field. This fact stimulated research for a new dynamical gauge theory of gravity. Reviews of such attempts can be found in Refs. [1], [2]. Also an overview of the geometrodynamics program applied to Yang-Mills theories and gravitational theories with torsion can be found in Ref. [3]. The Lagrangians of these theories contain terms which are quadratic in the curvature and/or torsion. Thus, in these theories the price for making the torsion dynamic is that one must introduce quadratic terms into the Lagrangian.

In this paper we attempt to make torsion a dynamical quantity, while leaving the EC Lagrangian unchanged. This is done by introducing an auxiliary classical spinor field. The EC field equations in this case give an algebraic relation between the torsion and spinor field that allow us to interpret the nonlinear Heisenberg equation for the spinor field as a dynamical equation for torsion [4]. The reverse connection that is made in this paper between spin and torsion gives a geometrization of spin. This idea (that spin should have a geometrical interpretation) goes back to Wheeler [5]. Along similar lines a Rainich type geometrization of spin has been carried out in Ref. [6].

\section{EINSTEIN-CARTAN GRAVITY WITH SPINOR FIELD}

The Lagrangian for EC gravity with a classical spinor field can be written as (in this section we follow the notation of Ref. 44):

$$
L=\frac{\hbar c}{2}\left[i \bar{\psi} \gamma^{\mu}\left(\nabla_{\mu} \psi\right)-\frac{m c}{\hbar} \bar{\psi} \psi+(\text { Hermitian }- \text { conjugate })\right]-\frac{1}{2 k} R,
$$


here Greek indices $=0,1,2,3$ are $4 \mathrm{D}$ spacetime indices; $\gamma^{\mu}$ are Dirac matrices satisfying $\left\{\gamma^{\mu}, \gamma^{\nu}\right\}=2 g^{\mu \nu} ; g^{\mu \nu}$ is the $4 \mathrm{D}$ spacetime metric; $k=8 \pi G / c^{4} ; R=g^{\beta \gamma} R_{\beta \alpha \gamma}^{\alpha}$ is the 4D Ricci scalar of the affine connection $\Gamma_{\bullet B C}^{A}$ (The definitions of the various Riemann-Cartan geometrical objects are given in Appendix A). A modern account of EC-Dirac theory with torsion is given in Ref. [7]. In Eq. (11) we have included the standard mass parameter, $m$. Heisenberg's original idea in his studies of the non-linear Dirac or Heisenberg equation was that one should set $m=0$, and that the mass should appear dynamically via $M=\int T_{00} d^{3} x$ ( $T_{\alpha \beta}$ is energy-momentum tensor for the spinor and electromagnetic fields). The covariant derivative of the spinor field is defined as [8], [9]:

$$
\nabla_{\mu} \psi=\left(\partial_{\mu}-\frac{1}{4} \omega_{a b \mu} \gamma^{[a} \gamma^{b]}-\frac{1}{4} S_{a b \mu} \gamma^{[a} \gamma^{b]}\right) \psi
$$

here $a, b=0,1,2,3$ are vier-bein indices; $\gamma^{a}=\gamma^{0}, \gamma^{1}, \gamma^{2}, \gamma^{3}$ are ordinary Dirac matrices $\left\{\gamma^{a}, \gamma^{b}\right\}=2 \eta^{a b} ; \gamma^{\mu}=h_{a}^{\bullet \mu} \gamma^{a}\left(h_{\bullet \mu}^{a}\right.$ is a vier-bein $) ; \eta^{a b}=\operatorname{diag}\{1,-1,-1,-1\}$ is the $4 \mathrm{D}$ Minkowski metric; [ ] means antisymmetrization. The coefficients of the spinor connection are defined as follows:

$$
\omega_{a b \mu}=h_{a \alpha} h_{b}^{\bullet \nu}\left\{\begin{array}{l}
\alpha \\
\mu \nu
\end{array}\right\}+h_{a}^{\bullet \nu} \frac{\partial h_{b \nu}}{\partial x^{\mu}}
$$

here $\left\{\begin{array}{l}\alpha \\ \mu \nu\end{array}\right\}$ are the Christoffel symbols. If the torsion is taken as totally antisymmetric then varying the torsion, spinor fields and metric leads to the following fields equations:

$$
\begin{gathered}
S^{a b c}=4 i l_{P l}^{2}\left(\bar{\psi} \gamma^{[a} \gamma^{b} \gamma^{c]} \psi\right), \\
\left(i \gamma^{\mu} \partial_{\mu}-\frac{i}{4} \omega_{a b \mu} \gamma^{\mu} \gamma^{[a} \gamma^{b]}+l_{P l}^{2}\left(\bar{\psi} \gamma^{[a} \gamma^{b} \gamma^{c]} \psi\right) \gamma_{[a} \gamma_{b} \gamma_{c]}-\frac{m c}{\hbar}\right) \psi=0, \\
R_{\mu \nu}-\frac{1}{2} g_{\mu \nu} R=8 l_{P l}^{2} T_{\mu \nu}^{D}-8 l_{P l}^{4} g_{\mu \nu}\left(\bar{\psi} \gamma^{[a} \gamma^{b} \gamma^{c]} \psi\right)\left(\bar{\psi} \gamma_{[a} \gamma_{b} \gamma_{c]} \psi\right),
\end{gathered}
$$

here $S^{a b c}$ is the antisymmetric torsion tensor, $l_{P l}=\sqrt{\pi \hbar G / c^{3}}$ is the Planck length, $R_{\mu \nu}$ is the Ricci tensor, $g_{\mu \nu}$ is the metric which is defined in the ordinary way $g_{\mu \nu}=h_{\bullet \mu}^{a} h_{a \nu}$. The energy-momentum tensor $T_{\mu \nu}^{D}$ of the Dirac field is:

$$
T_{\mu \nu}^{D}=\frac{i}{4}\left[\bar{\psi} \gamma_{\mu}(\stackrel{\{\}}{\nabla} \nu \psi)+\bar{\psi} \gamma_{\nu}\left(\nabla_{\mu} \psi\right)\right]+(\text { Hermitian - conjugate }),
$$

here $\stackrel{\{\}}{\nabla}_{\mu}$ means the covariant derivative without torsion. 


\section{DYNAMICAL TORSION FROM HEISENBERG EQUATION. EINSTEIN-CARTAN-HEISENBERG GRAVITY}

Eq.(4) establishes an algebraic connection between the torsion and the spinor field. Ordinarily the right hand side of this equation (the spinor field) is interpreted as the source of the left side (the torsion). We propose [4 the reverse interpretation: torsion is the source for the classical spinor field. In the $4 \mathrm{D}$ case the totally antisymmetric torsion can be repre-

sented as the pseudo-vector $S^{\mu}=\epsilon^{\mu \beta \gamma \delta} S_{\beta \gamma \delta}$ and has 4 independent degrees of freedom. The spinor $\psi$ also has 4 components. Hence the algebraic connection between $S^{\mu}$ and $\bar{\psi} \gamma^{\mu} \gamma^{5} \psi$ allows us to express the spinor components $\psi_{a}(a=1,2,3,4)$ in terms of the components of the torsion vector $S^{\mu}(\mu=0,1,2,3)$ and then to substitute this back into the Heisenberg equation (5). In this way the Heisenberg equation becomes a dynamical equation for torsion so that the torsion becomes a propagating field. This leads to an essential change of the physical interpretation of EC gravity coupled to a classical spinor field. Using the relationship between the spinor field and the torsion we can replace the spinor field in the Heisenberg equation by the torsion and view this theory as a pure vacuum gravitational theory with propagating torsion. In the rest of this paper we will refer to such a theory, where the torsion has become a propagating degree of freedom via the switch with the spinor field, as Einstein-Cartan-Heisenberg gravity. The Heisenberg equation (5) now becomes a dynamical equation for torsion which is of a form similar to the non-linear, spinor equations investigated by Ivanenko [10] and Heisenberg [11], [12].

\section{HEISENBERG GRAVITY}

We now want to examine the Einstein-Cartan-Heisenberg gravitational theory in the limit where the metric degrees of freedom are frozen out. Ref. [1] gives a classification of different gravity theories with curvature and/or torsion:

1. Riemann-Cartan space $U_{4}$ has both curvature and torsion. 
2. Weitzenböck space $W_{4}$ has only torsion; curvature $=0$.

3. Riemann space $V_{4}$ has only curvature; torsion $=0$.

4. Minkowski space $M_{4}$ has curvature $=$ torsion $=0$.

EC gravity is associated with $U_{4}$, and ordinary Einstein gravity is associated with $V_{4}$. The gravitational theory associated with $W_{4}$, Weitzenböck space, we will call Heisenberg gravity. With the connection between the spinor field and the torsion the Heisenberg equation for the spinor field becomes the gravitational equation for the torsion. The implicit form for this equation is

$$
\begin{gathered}
S^{a b c}=4 i l_{P l}^{2}\left(\bar{\psi} \gamma^{[a} \gamma^{b} \gamma^{c]} \psi\right) \\
\left(i \gamma^{\mu} \partial_{\mu}+l_{P l}^{2}\left(\bar{\psi} \gamma^{[a} \gamma^{b} \gamma^{c]} \psi\right) \gamma_{[a} \gamma_{b} \gamma_{c]}-\frac{m c}{\hbar}\right) \psi=0
\end{gathered}
$$

Now we examine two solutions in this theory.

\section{A. 4D Trivial solution}

We consider the constant spinor field in $4 \mathrm{D}$ Weitzenböck spacetime where $\omega_{a b \mu}=0$ and we further assume $m=0$ 円 :

$$
\psi(r, t)=\left\{\begin{array}{l}
a \\
b \\
c \\
d
\end{array}\right\},
$$

here $a, b, c, d$ are constants. The spin density vector $S^{\mu} \propto\left(\bar{\psi} \gamma^{\mu} \gamma^{5} \psi / i\right)$ (here we use $\gamma^{5}=$ $\left.\gamma^{0} \gamma^{1} \gamma^{2} \gamma^{3}\right)$ then takes on the following form:

\footnotetext{
${ }^{1}$ As mentioned in section 2 Heisenberg's viewpoint was that $m=0$ was the most natural condition, and the mass should be determined dynamically via the time-time part of the energy-momentum density
} 


$$
\begin{array}{r}
S^{0} \propto-2 \operatorname{Re}\left(\mathrm{ac}^{*}+\mathrm{bd}^{*}\right), \\
S^{1} \propto-2 \operatorname{Re}\left(a b^{*}+\mathrm{cd}^{*}\right), \\
S^{2} \propto 2 \operatorname{Im}\left(a b^{*}+\mathrm{cd}^{*}\right), \\
S^{3} \propto|a|^{2}-|b|^{2}+|c|^{2}-|d|^{2}
\end{array}
$$

Combining this with the Heisenberg equation (9) we find the following relationships between the constants

$$
\begin{gathered}
a|a|^{2}+a|b|^{2}-a|d|^{2}-a^{*} c^{2}+b c d^{*}-b^{*} c d=0, \\
|a|^{2} b+a c^{*} d-a^{*} c d+b^{*}|b|^{2}-b|c|^{2}-b^{*} d^{2}=0 \\
a^{2} c^{*}+a b d^{*}-a b^{*} d+|b|^{2} c-c|c|^{2}-c|d|^{2}=0, \\
|a|^{2} d+a b c^{*}-a^{*} b c+b^{2} d^{*}-|c|^{2} d-d|d|^{2}=0
\end{gathered}
$$

These equations have the following simple solution:

$$
a=b=c=d
$$

The simplicity of this solution is a result of the nonlinearity of the Heisenberg equation. This simple, constant solution is closely related to that found in Ref. [13]. The spin density is:

$$
S^{\mu} \propto\left\{|a|^{2} ;|a|^{2} ; 0 ; 0\right\}
$$

This indicates that the spin density is a constant vector in the $(t x)$ plane.

Another interesting aspect of this trivial solution is that it contains no field energymomentum in $\psi$. Using Eq. (10) in Eq. (7) immediately gives $T_{\mu \nu}^{D}=0$ since $\psi$ has neither time or spatial dependence.

\section{B. 5D Spherically symmetric solution}

It is straightfoward to generalize much of the 4D development to 5D Kaluza-Klein theory. In doing this we will work with a 5D Weitzenböck spacetime (i.e. with the metric degrees 
of freedom being frozen), and we will again take the torsion to be totally antisymmetric. In this way we find that the 5D Heisenberg equations are practically identical to the $4 \mathrm{D}$ Heisenberg equations. This allows us to take over some of the well known 4D, finite energy solutions [13] [14, and use them as solutions for the 5D Heisenberg equation. Varying the torsion and spinor fields leads to the following field equations which, except for the range of the indices, is similar to the $4 \mathrm{D}$ Heisenberg equations (5) and (9)

$$
\begin{gathered}
S^{a b c}=4 i l_{P l}^{2}\left(\bar{\psi} \gamma^{[a} \gamma^{b} \gamma^{c]} \psi\right), \\
\left(i \gamma^{A} \partial_{A}+l_{P l}^{2}\left(\bar{\psi} \gamma^{[a} \gamma^{b} \gamma^{c]} \psi\right) \gamma_{[a} \gamma_{b} \gamma_{c]}-\frac{m c}{\hbar}\right) \psi=0
\end{gathered}
$$

here $S^{a b c}$ is the 5D antisymmetric torsion tensor; $a, b, c=0,1,2,3,4$ are five-bein indices; $A=0,1,2,3,4$ is the 5D world index; $\left\{\gamma^{A}, \gamma^{B}\right\}=2 \eta^{A B} ; \eta^{A B}=\operatorname{diag}(1,-1,-1,-1,-1)$. The Dirac matrix $\gamma^{4}$ is defined to be $\gamma^{4} \equiv \gamma^{5}$. The ansatz for Eq. (22) is taken as the standard spherically symmetric spinor:

$$
\psi(r, t)=e^{-i \omega t}\left\{\begin{array}{c}
i f(r) \\
0 \\
g(r) \cos \theta \\
g(r) \sin \theta e^{i \varphi}
\end{array}\right\},
$$

here $r, \theta, \varphi$ are the spherical coordinates. The substitution of ansatz (23) into Heisenberg's Eq. (22) gives us the following two equations:

$$
\begin{array}{r}
g^{\prime}+(-m+\omega) f+\frac{2 g}{r}-12 l_{P l}^{2} f\left(f^{2}-g^{2}\right)=0 \\
f^{\prime}-(m+\omega) g-12 l_{P l}^{2} g\left(f^{2}-g^{2}\right)=0
\end{array}
$$

here $\hbar, c=1$. These equations of the 5D Heisenberg equation coincide identically with equations for the 4D Heisenberg equation, which have been investigated numerically in Ref. 14 with other nonlinear terms (e.g. $(\bar{\psi} \psi)^{2}$ and $\left.\left(\bar{\psi} \gamma^{\mu} \psi\right)^{2}\right)$. Exact solutions to the Heisenberg equation in a curved spacetime, and with the simplified interaction term $(\bar{\psi} \psi)^{2}$, were investigated in Ref. [13]. Since in the present work we have frozen out the metric 
degrees of freedom we will concentrate mainly on the solutions given in Ref. [14 for our 5D Kaluza-Klein system. Equations (24) - (25) have regular solutions in all space only for some discrete set of initial values $f_{n}(0)$ ( $n$ is the number of intersections that $f(r)$ makes with the $r$-axis). Near the origin the regular solution has the following behavior:

$$
\begin{gathered}
g(r)=g_{1} r+g_{3} \frac{r^{3}}{6}+\cdots \\
f(r)=f_{0}+f_{2} \frac{r^{2}}{2}+\cdots
\end{gathered}
$$

Substituting Eqs. (26) - 27) into Eqs (24) - (25) gives:

$$
\begin{gathered}
g_{1}=\frac{f_{0}}{3}\left[12 l_{P l}^{2} f_{0}^{2}+(m-\omega)\right], \\
f_{2}=g_{1}\left[12 l_{P l}^{2} f_{0}^{2}+(m+\omega)\right] .
\end{gathered}
$$

This means that for fixed $m$ and $\omega$ a solution depends only on the initial value $f(0)=f_{0}$. For arbitrary $f_{0}$ values the solution is singular at infinity $(r \rightarrow \infty)$. However there are a discrete series of $\left(f_{0}\right)_{n}$ values for which the solution is regular at infinity. A more detailed discussion of the properties of these solutions can be found in Ref. [14]. Thus, Eqs. (24) and (25) have a discrete spectrum of regular solutions in all space, and they have finite energy. At infinity $(r \rightarrow \infty)$ these solutions have the following asymptotic behavior:

$$
\begin{array}{r}
f=f_{\infty}+\frac{a e^{-\alpha r}}{r^{2}}+\cdots, \\
g=\frac{b e^{-\alpha r}}{r^{2}}+\cdots, \\
\alpha^{2}=4 \omega(m+\omega), \\
\frac{b}{a}=-\sqrt{1-\frac{m}{\omega}}, \\
f_{\infty}= \pm \sqrt{\frac{-m+\omega}{12 l_{P l}^{2}} .}
\end{array}
$$

This guarantees the finiteness of all the physical parameters. As an example the field energy density, $T_{00}$, of this solution can be finite. Inserting the general form of the spinor from Eq. (23) into the energy-momentum tensor of Eq. (17) gives

$$
T_{00}^{D}=\omega\left(f^{2}+g^{2}\right)
$$


This will give a finite energy when integrated over all space if the ansatz function $f(r), g(r)$ fall off rapidly enough as $r \rightarrow \infty$. From the asymptotic form of these functions given in Eqs. (30) - (34), this will occur if $f_{\infty}=0$, which in turn implies the condition $m=\omega$. One can interpret the finite integral of the energy density over all space as the mass of the solution (i.e. $\left.M=\int T_{00}^{D} d^{3} x\right)$.

We can consider 5D Kaluza-Klein spacetime as the principal bundle over 4D Einstein spacetime. In this case (at least locally) 5D spacetime, which is the total space of the principal bundle, is $M_{5}=M_{4} \times U(1)$, where $M_{4}$ is an ordinary 4D Einstein spacetime (the base of the principal bundle) and $U(1)$ is the electromagnetic gauge group (the fibre of the principal bundle). We note that in this case the spacetime directions along the $M_{4}$ base and $U(1)$ fibre are not equivalent. This is very easy to understand: the points of the base $M_{4}$ are the ordinary spacetime points and the $M_{4}$ curvature can change from point to point, but the points on the $U(1)$ fibre are the elements of the gauge group and hence the curvature of the fibre is constant (it can depend only on spacetime points of the base). From a $4 \mathrm{D}$ observer's point of view we can introduce the 4D spin vector $S^{\mu} \propto\left(\bar{\psi} \gamma^{\mu} \gamma^{5} \psi\right)$ which for the ansatz of Eq. (23) becomes

$$
S^{\mu} \propto\left\{0 ;-g^{2} \sin 2 \theta \cos \varphi ;-g^{2} \sin 2 \theta \sin \varphi ;-f^{2}-g^{2} \cos 2 \theta\right\}
$$

This is the spin density from pure vacuum gravity, without any matter field. In this paper, after freezing out the metric degrees of freedom, we are left with only torsion. This torsion is interpreted as the source of the classical, auxiliary spinor field $\psi$, in both $4 \mathrm{D}$ and $5 \mathrm{D}$ versions of the theory. This is an inversion of the usual interpretation where the spinor is considered as fundamental and acts as the source for the torsion.

In Ref. [15] solutions for the nonlinear Heisenberg equation were obtained which in contrast to our localized nonsingular solution have the form of elliptic functions. 


\section{PHYSICAL DISCUSSION}

In this paper we considered 4D and 5D Heisenberg gravity, where the only dynamical, gravitational degree of freedom was the torsion. Usually torsion is a non-propagating, non-

dynamical degree of freedom. Here the dynamical behavior of the torsion is defined by the nonlinear Heisenberg equation (Eqs. (5) , (9) or (22)) through an auxiliary classical spinor field (it is possible that this field has some independent physical meaning but this is a problem for a future investigation). A nice feature of this dynamical torsion theory is that it possesses some simple, non-singular solutions (e.g. Eqs. (30) - (34)) which may yield a physical geometric model for spin. In contrast for Einstein gravity, where the torsion $=0$, the spherically symmetric Schwarzschild solution, while thought to give a good description of certain physical situations such as supermassive collapsed stars, nevertheless possesses unphysical singularities. This absence of singularities for these solutions of Heisenberg gravity may imply that the full Einstein-Cartan-Heisenberg gravity may also have singularity free solutions as compared to ordinary Einstein gravity. The main point that we want to emphasize however is that Heisenberg gravity appears to give a purely gravitational model for spin (the torsion is the source of the auxiliary classical spinor field).

Gravity can be formulated in terms of connections and metrics, with the torsion as part of the connection. In this paper we restricted ourselves to only dealing with the torsion by considering Weitzenböck space where curvature $=0$ so that the metric degrees of freedom are frozen out. Taking into account that the torsion is related to a bilinear combination of an auxiliary spinor field would then lead to a quantized version of the torsion via the quantization of the quadratic form of the auxiliary spinor field. The apparent problem with this is that the original Heisenberg system is hard to quantize by conventional means. In fact because of the non-linear fermion interaction term this theory is conventionally non-renormalizable. However, in the 50's Heisenberg [11], [12] proposed a nonperturbative method of quantizing the nonlinear Heisenberg equation. By applying this nonperturbative method, outlined in Ref. [11] [12], to the auxiliary spinor field it is hoped that the quanti- 
zation of torsion (=part of gravity) can be achieved through its connection to the auxiliary spinor field. Even if such a program of quantizing the torsion part of gravity is possible, this still leaves open the much harder and deeper question of how to quantize in Riemann or Riemann-Cartan space.

Another possible problem related to the quantization of the essentially classical theory outlined here, is that quantum effects could alter the connection between the torsion and the auxiliary spinor field given in Eq. (4) (or more directly quantum effects the could alter the connection given by $\left.S_{\mu} \propto \bar{\psi} \gamma^{\mu} \gamma^{5} \psi\right)$. In particular one may worry that this relationship could be effected by the axial anomaly [16]. Since the axial anomaly in this context would be connected with the metric degrees of freedom, the problem does not become an issue in our simplified model where these degrees of freedom have been frozen out. This possible problem would become important once one moved from Weitzenböck space to Riemann-Cartan space.

The ideas presented here have their origins in the attempts by Wheeler, Einstein and others to geometrize physics. As an example one can point to Wheeler's geometrical model of electrical charge as a wormhole threaded by electric flux (This "charge without charge" idea can be found in Ref. [5]). But this model can not be a realistic model for a charged fermion such as the electron since it does not have a spin angular momentum. Wheeler wrote in Ref. [5]: “... It is impossible to accept any description of elementary particles that does not have a place for spin $\frac{1}{2}$. ... Unless and until an answer is forthcoming, pure quantum geometrodynamics must be judged deficient as a basis for elementary particle physics ...." As outlined in this paper it is possible that Heisenberg gravity can give such a geometrical model of spin. In this case the spherically symmetric solution of Eqs. (30) - (34) would represent a pure geometrical model of the spin. In Ref. [17] a composite wormhole model of electrical charge was advanced in spirit of Wheeler idea of "charge without charge". Here by examining a gravity theory with torsion and the metric degrees of freedom frozen out (Heisenberg gravity) we arrive at a geometrical model for spin. Curiously in both the model of electric charge of Ref. [17] and the model for spin of the present paper it appears that a higher dimensional manifold (i.e. 5D) is required. The eventual hope is that in the full 
Einstein-Cartan-Heisenberg gravity one may find a wormhole with spin and charge ("charge without charge", "mass without mass" and "spin without spin"). Such a solution for the full ECH gravity would support the supposition of Einstein and Wheeler that Nature consists from nothing?

\section{ACKNOWLEDGMENTS}

VD is grateful to Georg Forster Research Fellowship of Alexander von Humboldt Foundation for financial support of this project.

\section{APPENDIX A: RIEMANN-CARTAN GEOMETRY}

Here we give a simple introduction to Riemann-Cartan geometry following Ref. [9]. The affine connection of Riemann-Cartan spacetime is:

$$
\Gamma_{\bullet B C}^{A}=\left\{\begin{array}{l}
B \\
A C
\end{array}\right\}+S_{B C \bullet}^{A}-S_{C \bullet B}^{A}+S_{\bullet A B}^{A},
$$

here $\left\{\begin{array}{l}B \\ A C\end{array}\right\}$ are Christoffel symbols. Cartan's torsion tensor $S_{B C}{ }^{A}$ is defined according to :

$$
S_{B C \bullet}^{A}=S_{\bullet B C}^{A}=\frac{1}{2} \Gamma_{[B C]}^{A}=\frac{1}{2}\left(\Gamma_{\bullet B C}^{A}-\Gamma_{\bullet C B}^{A}\right)
$$

The contorsion tensor is:

$$
K_{B C \bullet}^{A}=S_{B C \bullet}^{A}+S_{C \bullet B}^{A}-S_{\bullet A B}^{A}
$$

In this case the affine connection is:

$$
\Gamma_{\bullet B C}^{A}=\left\{\begin{array}{l}
B \\
A C
\end{array}\right\}-K_{B C}^{A} \bullet
$$

The Riemann curvature tensor is defined in the usual way as:

$$
\begin{gathered}
\quad R_{\bullet B C D}^{A}=\partial_{C} \Gamma_{\bullet B D}^{A}-\partial_{D} \Gamma_{\bullet B C}^{A}+\Gamma_{\bullet E C}^{A} \Gamma_{\bullet B D}^{E}-\Gamma_{\bullet E D}^{A} \Gamma_{\bullet B C}^{E}= \\
R^{\{\}}{ }_{\bullet B C D}^{A}+\nabla_{D} K_{\bullet B C}^{A}-\nabla_{C} K_{\bullet B D}^{A}+K_{\bullet E C}^{A} K_{\bullet B D}^{E}-K_{\bullet E D}^{A} K_{\bullet B C}^{E}
\end{gathered}
$$


A modified torsion tensor is:

$$
T_{B C}^{A}{ }^{A}=S_{B C}{ }^{A}+\delta_{B}^{A} S_{C D}{ }_{\bullet}^{D}-\delta_{C}^{A} S_{B D}{ }_{\bullet}^{D} .
$$

We can decompose the curvature scalar into Riemannian and contorsion pieces as follows:

$$
R=\stackrel{\{\}}{R}^{R}+2 \stackrel{\{\}}{\nabla}_{A}\left(K_{B}^{\bullet A B}\right)-T_{A}^{\bullet B C} K_{C B}{ }_{\bullet}^{A}
$$

For antisymmetric torsion we can write:

$$
\begin{array}{r}
S_{A B C}=T_{A B C} ; \quad K_{A B C}=-S_{A B C}, \\
R=\stackrel{\{\}}{R}-S_{A B C} S^{A B C} .
\end{array}
$$




\section{REFERENCES}

[1] F.W.Hehl, J.Dermott McCrea, E.W. Mielke, Y. Neeman, Phys. Rept., 258, 1 (1995).

[2] F.W.Hehl, P. von der Heyde and G.D. Kerlick, Rev. Mod. Phys., 48, 393 (1976).

[3] E.W. Mielke, Geometrodynamics of Gauge Fields - On the geometry of Yang-Mills and gravitational gauge theories, (Akademie-Verlag, Berlin 1987)

[4] V.Dzhunushaliev, Int. J. Mod. Phys., D7, 909 (1998).

[5] J.A.Wheeler, Geometrodynamics, (Academic Press New-York and London, 1962).

[6] K. Kuchar, Acta. Phys. Pol. 28, 695 (1965)

[7] E.W. Mielke, et. al., "Yang-Mills-Clifford form of the Einstein action", in : Gravity, Particles and Space-time, ed. P. Pronin and G. Sardanashvily (World Scientific, Singapore, 1996) p. $217-254$

[8] V. de Sabbata and C. Sivaram, Spin and Torsion in Gravitation, (World Scientific Publishing, 1994).

[9] F.W.Hehl and B.K.Datta, J.Math. Phys., 12, 1334 (1971).

[10] D.Ivanenko, Phys. Z. Sowjetunion, 13, 141 (1938).

[11] W. Heisenberg, Nachr. Akad. Wiss. Göttingen, N8, 111 (1953); W. Heisenberg, Z. Naturforsch., 9a, 292 (1954); W. Heisenberg, F. Kortel and H. Mütter, Z. Naturforsch., 10a, 425 (1955); W. Heisenberg, Z. für Phys., 144, 1 (1956); P. Askali and W. Heisenberg, Z. Naturforsc., 12a, 177 (1957); W. Heisenberg, Nucl. Phys., 4, 532 (1957); W. Heisenberg, Rev. Mod. Phys., 29, 269 (1957)

[12] W.Heisenberg, Introduction to the unified field theory of elementary particles., (Wiley, London, 1966).

[13] E.W. Mielke, J. Math. Phys., 22, 2034 (1981) 
[14] R. Finkelstein, R.Lelevier and M. Ruderman, Phys. Rev., 83, 326 (1951); R. Finkelstein, C. Fronsdal and P.Kaus, Phys. Rev., 103, 1571 (1956).

[15] D.Kurdgelaidze, JETP, 32, 1156 (1957); 34, 1587 (1958); 36, 842 (1959) (in Russian).

[16] Y. Obukhov et. al., Found. Phys. 27, 1221 (1997); E.W. Mielke and D. Kreimer, Int. J. Mod. Phys. D7, 535 (1998)

[17] V.Dzhunushaliev, "Multidimensional geometrical model of the renormalized electrical charge with splitting off the extra coordinates", awarded Honorable Mention by Grav. Res. Found., 1998; Mod. Phys. Lett.A, 13, 2179 (1998). 